\title{
Modeling and Simulation of Cascading Failures in Transportation Systems during Hurricane Evacuations
}

\author{
Yuan Zhu ${ }^{1},{ }^{1}$ Kaan Ozbay, ${ }^{2}$ Hong Yang, ${ }^{3}$ Fan Zuo, ${ }^{2}$ and Di Sha ${ }^{2}$ \\ ${ }^{1}$ Inner Mongolia Center for Transportation Research, Inner Mongolia University, \\ Rm A357c, Transportation Building, Inner Mongolia University South Campus, 49 S Xilin Rd, Hohhot 010020, \\ Inner Mongolia, China \\ ${ }^{2}$ Department of Civil and Urban Engineering \& Center for Urban Science and Progress (CUSP), Tandon School of Engineering, \\ New York University (NYU), 15 MetroTech Center, 6th Floor, Brooklyn 11201, NY, USA \\ ${ }^{3}$ Department of Modeling, Simulation \& Visualization Engineering, Old Dominion University, 4700 Elkhorn Ave, Norfolk 23529, \\ $V A, U S A$
}

Correspondence should be addressed to Yuan Zhu; zhuyuan@imu.edu.cn

Received 5 January 2021; Revised 25 March 2021; Accepted 13 April 2021; Published 23 April 2021

Academic Editor: Indrajit Ghosh

Copyright (c) 2021 Yuan Zhu et al. This is an open access article distributed under the Creative Commons Attribution License, which permits unrestricted use, distribution, and reproduction in any medium, provided the original work is properly cited.

\begin{abstract}
Effective and timely evacuation is critical in alleviating the impact of hurricanes. As such, evacuation models are often sought to support the preparedness of evacuations. One important task in the modeling process is to evaluate exogenous factors that cause transportation system capacity loss during evacuation. Typical factors include direct damage to the roadway network due to storm surge and cascading impacts because of other facilities failures. For example, power outage can lead to signal failure and subway suspension. This paper aims to develop a macroscopic simulation-based approach to study the capacity loss of the roadway network in evacuation due to signal loss as a consequence of power outage. In particular, to simulate the case in which traffic signals lose power, a capacity-reduction model from signalized intersections to unsignalized (all-way stop control) intersections was developed and calibrated using microscopic model created in SUMO and Synchro. We used the downtown Manhattan as a case study area and created a hypothetical powergrid network in terms of neighborhoods. Six scenarios were built to simulate power loss of different neighborhoods. The simulation results give insights on how cascading failures of power network affect roadway network and evacuation process.
\end{abstract}

\section{Introduction}

One of the most challenging issues in prehurricane evacuation planning is the uncertainties of the actual conditions that may cause roadway capacity loss and additional delays. So, evaluating such uncertainties is critical for emergency planning and management and, to some extent, to alleviate negative impacts of hurricanes. Road network degradation can be attributable to either endogenous or exogenous reasons. Endogenous risks refer to uncertainties of the transportation system supply, as discussed in Li and Ozbay [1], while exogenous risks are due to external impacts, specifically, incident-induced capacity loss during the evacuation operations. According to real-world incident data collected during evacuation before the landfall of Hurricane Sandy, there are five types of incidents that have a direct impact on the highway network: accident, debris, disabled vehicle, downed trees, and flooding $[2,3]$. Apart from direct impact of storm surge on the transportation network, hurricanes also bring disruptions to other city infrastructures, such as power and communication systems. Unlike accidents or flooding that directly results in lane/road blockages, these infrastructures do not direct impact on the evacuation routes. Instead, the strong wind and rain fall before hurricane landfall could result in downed trees and other incident that cause failure of power infrastructure and then cause cascading failures of the transportation systems. For example, massive power outages caused by hurricanes 
may take out traffic signals and result in gridlocks at the signalized intersections. Failure of the power system can also affect other transportation systems (e.g., subways) that heavily rely on power supply.

Existing research has made great efforts in modeling the impact of endogenous risks associated with transportation system planning in evacuation [1]. However, there was still limited progress in probing the impact of the exogenous risks. As such, this paper contributes to the literature by making some attempts in this perspective. Specifically, it aims to model the cascading failures of power networks during storm surge. In particular, it considered downtown Manhattan that has experienced severe cascading failures of infrastructure systems during Hurricane Sandy as the study area. Considering the limited availability of power-grid data, a hypothetical power network using urban neighborhoods as the fundamental units is constructed. Each neighborhood is denoted as a node, and hypothetical links are used to connect these nodes based on an adjacency matrix. A simulationbased approach is then developed to analyze different scenarios of traffic signal control system failures as a result of the power loss. This facilitates the evaluation of the cascading impact on evacuation performance and transportation system resiliency.

1.1. Literature Reviews. In recent years, cascading failures of various infrastructures have drawn increased attention of researchers. Fotouhi et al. [4] quantified the resilience of the urban traffic-electric power coupled system using mixedinteger program to address the interdependencies between urban traffic and power distribution systems. Their study provides an alternative perspective of power to evaluate the lifeline performance. Zhang et al. [5] proposed a cascading failure model of public transit network to evaluate station frequency density under deliberate attack of traffic signals. They built simulation experiments using Jinan in China as a background city to verify the feasibility of their model. Their results reveal that by optimizing the station intensity and bus lanes, cascading survivability of public transit network can be improved. Oricchio et al. [6] explored the signal operations in flash mode due to power loss. The key findings of their study show that flashing modes provide reasonable performance with lower demand but cause breakdowns at higher volumes.

Regardless of hurricane impact, a number of studies focus on methods to estimate the capacity of unsignalized intersections. Wegmann [7] proposed a methodology to estimate the capacity of unsignalized intersections using gap distributions and bunching of major road traffic, with nonhomogeneous and inconsistent behavior of minor road traffic. Baass [8] pointed out that a number of vehicles that can cross an intersection depend on two factors: number of acceptable gaps and gap-acceptance distributions. Brilon and Miltner [9] discussed the conflicts of stop-control intersections and proposed the "Conflict Technique" to quantify the capacity loss for the unsignalized intersections. Prasetijo et al. [10] developed a regression method to measure unsignalized capacity based on the conflicting streams with distinct average speed and flow assigned to each stream. The estimated values were validated by empirical data from Indonesia. Another methodology based on evaluating expected average delays at multimodal uncontrolled intersections was presented in [11], which can represent the overall magnitude of capacity and delay. However, most of the previous research regarding capacity measurements of stop-controlled intersections is based on microscopic scenarios. Very few studies have considered the capacity loss under hurricane scenarios [2]. To utilize macroscopic planning model to quantify capacity reduction under signal failures, a new and more generalized approach is needed.

1.2. Data and Modeling Tools. The network model is based on the second generation of New York Best Practice (NYBPM 2G) [12] network. It is a widely used macroscopic planning model based on TransCAD 6.0 [13]. The NYBPM network is a generalization of the street network of New York City (NYC) metropolitan area that can be regarded as a directed graph. The intersections and roadways between intersections are represented by nodes and links of the graph, respectively. Each link also has attributes, such as from-node, to-node, direction (s), number of lanes per direction, functional class (fclass [14] and codes shown in Table 1), and original capacity. There is no attribute for nodes, but their attributes can be retrieved by adjacent links and combined with other spatial data. The nodes and links do not completely overlap with (but close to) actual roads and intersections, and some of local streets are replaced by centroid connectors. Most of major streets, especially for Manhattan, are kept in the model.

The limitation of this network is that there is no information on whether an intersection is signalized or not. To solve this issue, OpenStreetMap (OSM) [15] data are used. The OSM data contain the information of signalized intersections, such as signal post location and base signal timings. These attributes can provide the signalization information of all the intersections in the studied network.

To estimate and calibrate the intersection capacity under the power-loss scenario, two distinct microscopic simulation tools, namely, Behrisch [16] and Husch [17], are utilized. SUMO is a robust microsimulation tool that gained popularity in recent years, due to the flexibility of scenario settings, including detailed capabilities of simulation of signalized and unsignalized intersections. Synchro is a traditional microscopic simulation tool which is widely used in the US. Synchro includes a powerful signal-timing optimization tool and HCM 2010 [18] support, which can be applied to measure the performance of intersections under varied control scenarios. Particularly, the unsignalized intersection delay model was included in the tool, as mentioned in Chapter 19 and 20 of HCM 2010. Both simulation tools can estimate the capacity reduction due to the transition from signalized to unsignalized control, by measuring saturation flow rates under two circumstances where their level of services (LOS) are assumed to be equivalent. 
TABLe 1: Functional class codes [14].

\begin{tabular}{lccc}
\hline Type & Urban code & Rural code & Code for modeling \\
\hline Principal arterial-interstate & 11 & 01 & 1 \\
Principal arterial-other freeway/expressway & 12 & 02 & 2 \\
Principal arterial-other & 14 & 04 & 3 \\
Minor arterial & 16 & 06 & 4 \\
Major collector & 17 & 07 & 5 \\
Minor collector & 18 & 08 & 6 \\
Local & 19 & 09 & 7 \\
\hline
\end{tabular}

\section{Methodology}

This main objective of this paper is to build and test an evacuation scenario under the cascading failures of power and signalized intersection networks. The lower Manhattan in New York City is selected as the study area. Before and after the land fall of Hurricane Sandy, the study area suffered from significant power-loss and signal failures that lasted for a couple of days.

Typically, malfunction of traffic signals caused by power outage shows flashing red lights for several hours using their backup power and then becomes dark if the outage lasts longer. In the United States, a flashing red is equivalent to all-way stop control (AWSC) [18]. Therefore, this study assumes that the control type of intersections converts from signalized to AWSC when they lose power, which is the needed safety action when traffic signals encounter outage. In the macroscopic model, the effect of the switch from a signalized to an unsignalized intersection can be measured by calculating the difference between the capacity of a signalized capacity and the capacity of AWSC. So, the core of this modeling methodology is how to quantify the loss of intersection capacity as a result of power outage.

There are some important challenges. Firstly, in typical modeling of AWSC intersections, input like turning movements or turning rates are known. However, they are absent in the data used in this study. Secondly, the signal timing of the NYBPM model is considered through the reduction of intersection capacity and cannot be extracted separately. Thus, it is not possible to build a relationship between existing signalized and unsignalized intersection capacities. Considering intersection types, directions, and number of lanes, there are more than a hundred combinations of intersection structures in the study area, including four-way and $\mathrm{T}$ ( or Y) intersections. A number of lanes for each direction range from 0 (one-way street) to 5. HCM 2010 provides a method to calculate the capacity of the AWSC intersections. However, such method does not include the cases in which the number of lanes per direction exceeds two (such case satisfies the warrants of signalized intersection). The same limitation also applies to Synchro. SUMO, however, can simulate unsignalized scenarios with more than two lanes, but its capacity is related to a number of vehicular parameters such as gap acceptance and driver aggressiveness. These values can be adjusted to identify capacity values that are in agreement with the Synchro output.

By considering aforementioned difficulties, a generalized solution based on microsimulation models of selected typical intersections can be built. Both in Synchro and SUMO, intersections with no more than three lanes per direction are created. The capacity value obtained from SUMO is calibrated using Synchro results. For intersections with more than three lanes, capacity is calculated based on SUMO's estimations. Finally, the capacity along with attributes of selected intersections is used to build a predictive model of unsignalized capacity.

Following sections describe the modeling approach in detail. Firstly, the data for modeling and prediction are filtered and processed. Then, capacity-loss models are constructed based on results from microscopic simulation models. Lastly, the model is used to predict capacity loss for all signalized intersections and update the simulation network.

2.1. Data Filtering and Processing. As mentioned above, there are no attributes for nodes, so spatial attributes, such as neighborhood tabulation area (NTA) that intersection belongs to, are assigned to the nodes. Besides, attributes of connected links can also be found by using a lookup table of links. This step constructs a data table of nodes that contains information about connected links such as name of the streets, number of lanes, and capacity for each direction.

The following step is used to filter the nodes that correspond to signalized intersections out of all nodes. This approach contains two operations: check if the node is actually an intersection (not expressway interchange or ramp), and if they are close to traffic signals. This can be done as follows: if all the links connect to the node belong to an expressway (whose fclass is 11 or 12), the node is not an intersection. If the node is confirmed as an intersection, the distance from each node to the location of nearest traffic light is measured, which is obtained from OSM data. If the distance is lower than certain value, the node is identified as signalized. In this study, 220 feet is selected as the filtering threshold. The filtered signalized intersection is shown in green dots in Figure 1.

Another task for data processing is to determine the number of conflicts for each intersection. According to HCM 2010, when the intersection is operating as AWSC, the capacity of approach is related to conflicting approaches, as shown in Figure 2. If traffic is present in the study approach only (Case 1), subject vehicles depart as rapidly as individual drivers can safely accelerate and clear the intersection. If one or more vehicles present in opposing approaches (Cases 2 to 5), subject vehicles have to yield to other approaches to make 


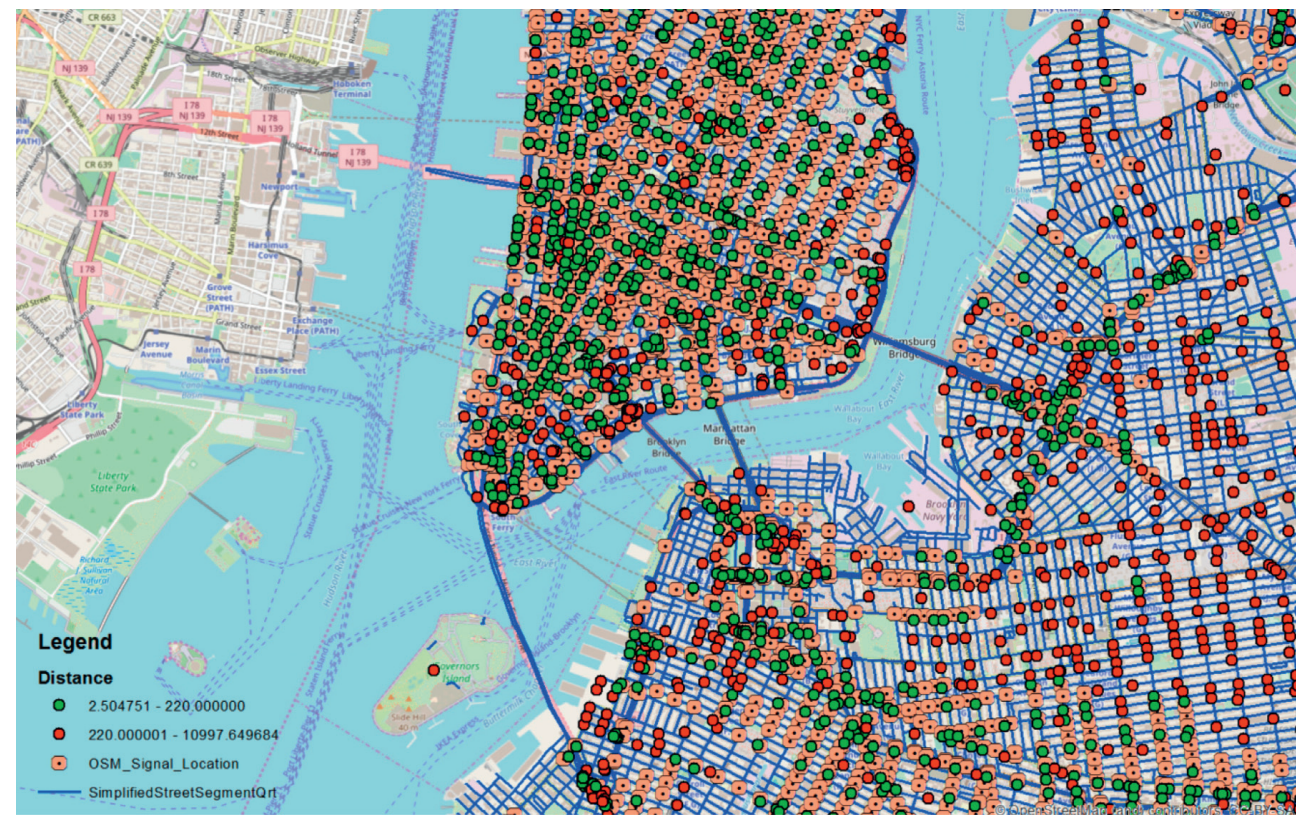

FIGURE 1: Filtered signalized intersections.

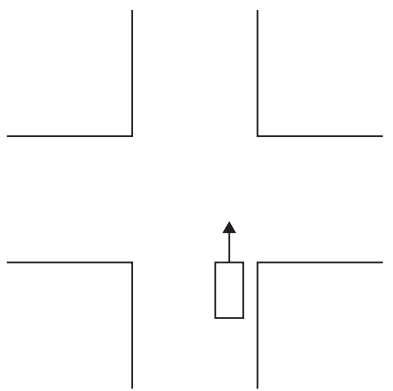

Case 1: vehicle (s) on subject approach only
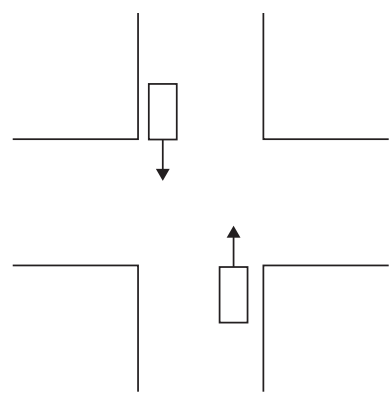

Case 2: vehicles on subject and opposing approaches
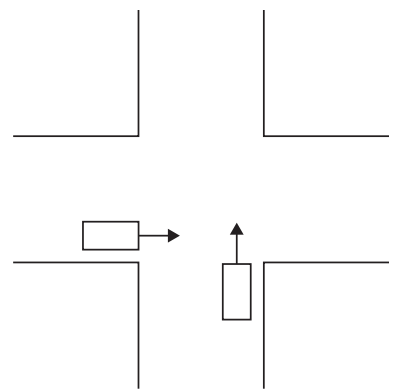

Case 3: vehicles on subject and conflicting approaches

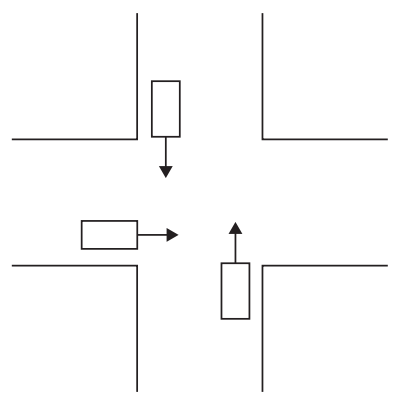

Case 4: vehicles on subject and two other approaches

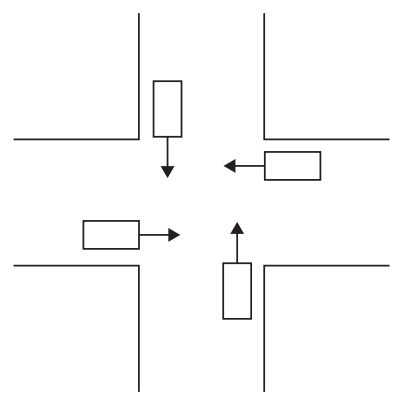

Case 5: vehicles on all approaches

FIGURE 2: Description of opposing approaches.

sure that only one vehicle can pass the intersection at the same time. It can be implied that the throughput of AWSC intersection is directly related to number of opposing approaches or number of conflicts, which can be calculated from intersection attributes. For example, if there are four links connected to a node $A$ and three links have lanes towards $A$, the number of conflicts for node $A$ is 2 . For T and four-way intersections, the maximum numbers of conflicts are 2 and 3, respectively. Please refer to Figure 3 for different types of conflicts. The data filtering and processing algorithm is summarized in Figure 4.

2.2. Modeling of Intersection Capacity Loss. As mentioned above, Synchro and SUMO are used to calculate and calibrate the unsignalized intersection capacity loss. Five intersections 


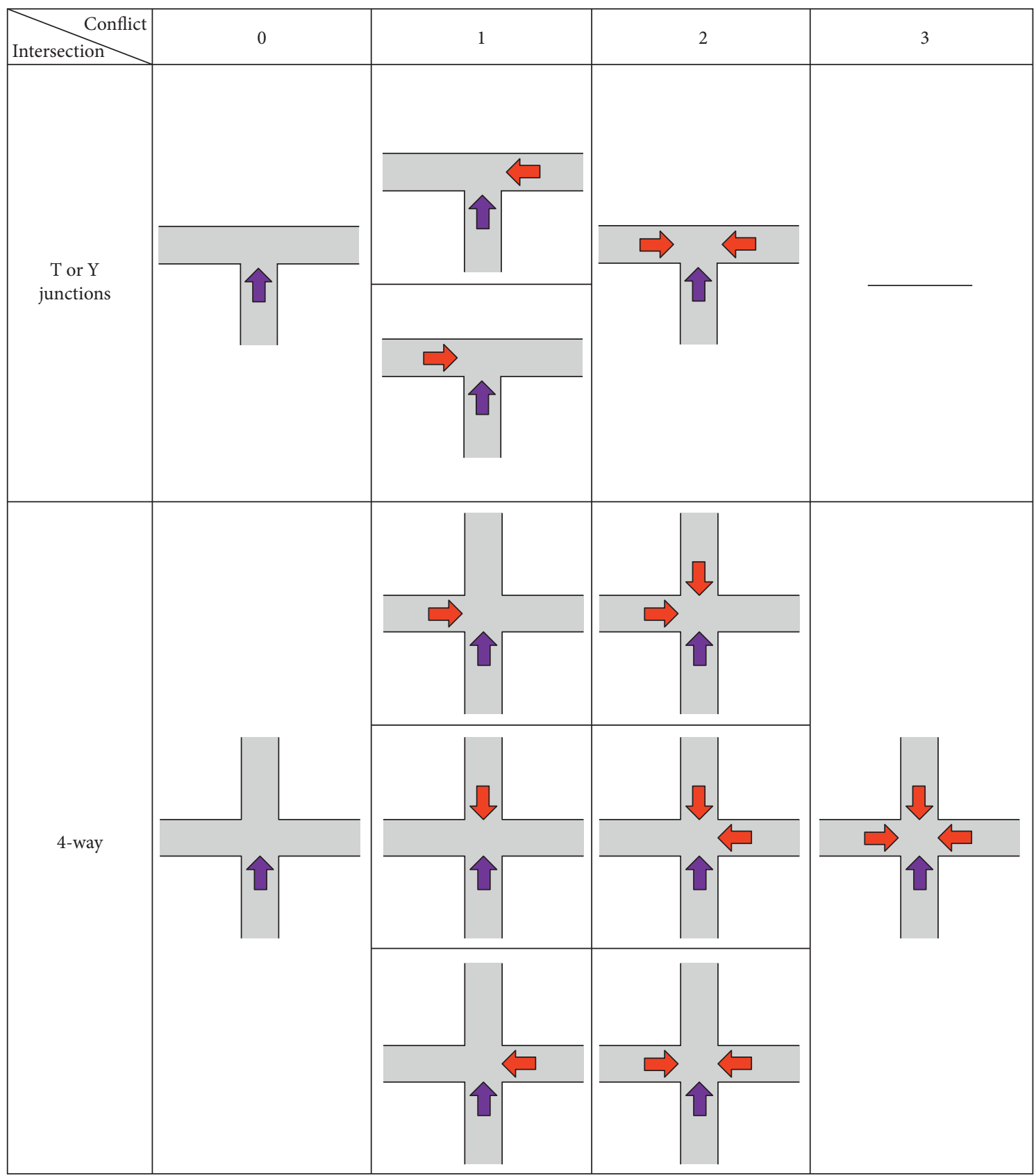

Figure 3: Conflict numbers and types.

located in lower Manhattan are selected as the examples. The attributes of selected intersections are shown in Table 2.

In the first step, Synchro models are used to measure the capacity of intersections based on default parameters defined in HCM 2010. Taking Synchro capacity as a benchmark, multiple simulation runs are made in SUMO. In each run, crucial behavioral parameters such as gap acceptance and acceleration rates are adjusted so that the output saturation flow rate approaches the Synchro capacity. Table 3 shows calibrated SUMO output based on Synchro capacity.

Based on the calibrated SUMO model, capacity of other intersections is simulated. Output capacity of intersections is illustrated in Table 4, along with attributes of the links.
It is observed that the capacity shows strong linear correlation with the attributes of intersections. Thus, they were applied to all the intersections of the similar types in the studied network.

2.3. Prediction and Assignment of Capacity Loss. The last step of this methodology is to estimate the capacity loss, by reducing the capacity to the unsignalized level for all signalized intersections in the designated area. This is implemented by using the lookup table for all intersections within the cascading failure zones, and we predict the capacity loss for each relevant link. It should be noted that 


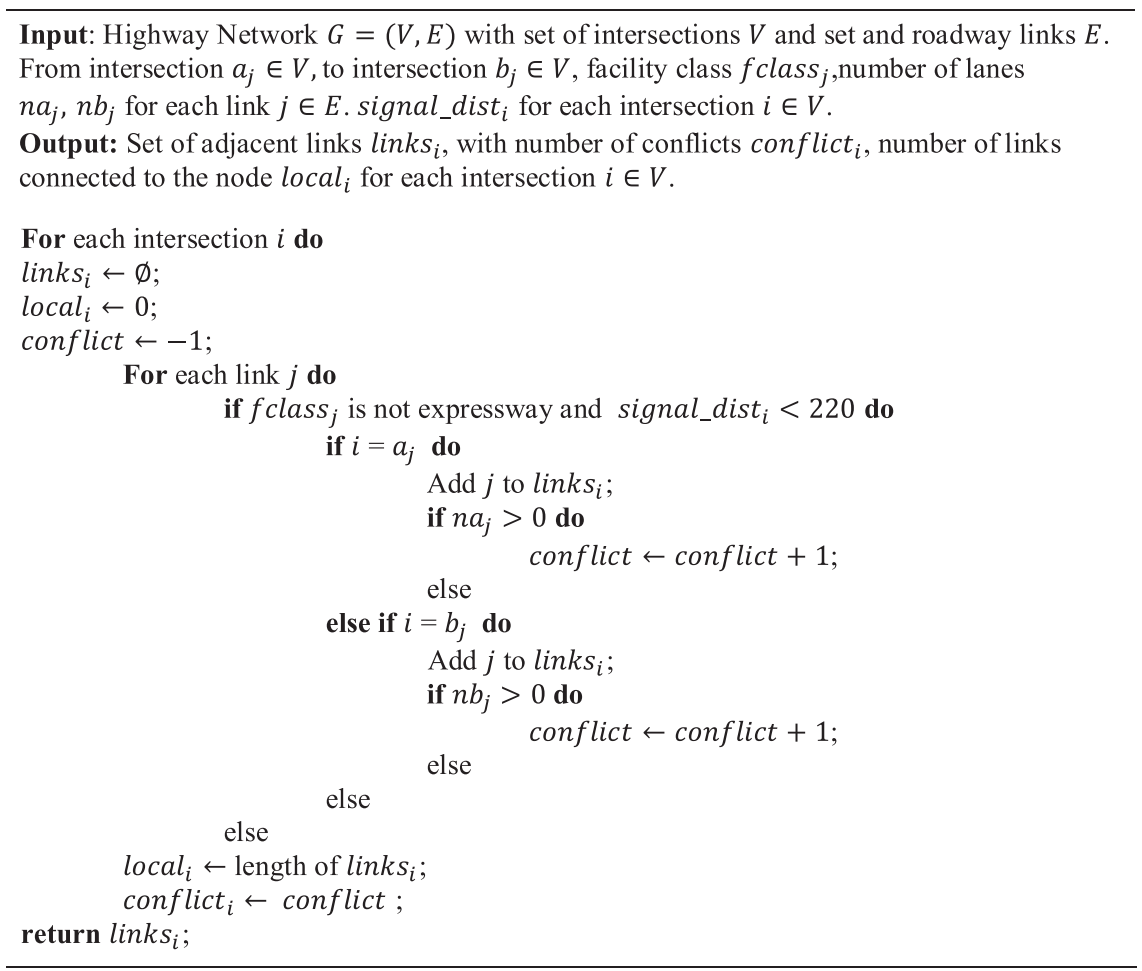

FIgUre 4: Pseudocodes of data filtering and processing.

TABLE 2: Selected intersections and attributes.

\begin{tabular}{|c|c|c|c|}
\hline Intersection & Approaches & Lanes & Conflicts \\
\hline \multirow{3}{*}{ Broadway and Canal Street } & Broadway SB & 4 & \multirow{3}{*}{2} \\
\hline & Canal WB & 3 & \\
\hline & Canal EB & 3 & \\
\hline \multirow{2}{*}{ Center Street and Hester Street } & Center NB & 3 & \multirow[b]{2}{*}{1} \\
\hline & Hester WB & 1 & \\
\hline \multirow{2}{*}{ Lafayette Street and Walker Street } & Lafayette SB & 2 & \multirow{2}{*}{1} \\
\hline & Walker EB & 1 & \\
\hline \multirow{2}{*}{ Broadway and Franklin Street } & Broadway SB & 2 & \multirow{2}{*}{1} \\
\hline & Franklin EB & 1 & \\
\hline \multirow{2}{*}{ Church Street and Walker Street } & Church NB & 3 & \multirow{2}{*}{1} \\
\hline & Walker EB & 1 & \\
\hline
\end{tabular}

TABLE 3: Calibration results of selected intersection.

\begin{tabular}{|c|c|c|c|c|c|}
\hline Intersection & Approaches & Synchro & SUMO & Delay $(s)$ & $\mathrm{V} / \mathrm{C}$ ratio \\
\hline \multirow{3}{*}{ Broadway and Franklin Street } & Broadway (T) & 804 & \multirow[t]{2}{*}{893} & \multirow[t]{2}{*}{44.7} & \multirow[t]{2}{*}{0.98} \\
\hline & Broadway (L) & 89 & & & \\
\hline & $\begin{array}{c}\text { Franklin (T) } \\
\text { Franklin I }\end{array}$ & $\begin{array}{c}416 \\
32\end{array}$ & 448 & 43.8 & 0.98 \\
\hline
\end{tabular}

the lower bound of reduced capacity (if original capacity is greater than 0 ) is set to 100 rather than 0 . The reason for this assumption is for computational needs that allow iterations of traffic assignment to reach convergence eventually.
Finally, capacity-loss table for each scenario is built and fed into the evacuation assignment model. The procedure adopted in this step is summarized as a pseudocode shown in Figure 5. 
TABLE 4: Modeling parameters.

\begin{tabular}{|c|c|c|c|c|}
\hline Intersection & Lanes & Conflicts & fclass & Capacity \\
\hline \multirow{3}{*}{ Broadway and Canal Street } & 4 & 2 & 14 & 866 \\
\hline & 3 & 2 & 14 & 639 \\
\hline & 3 & 2 & 14 & 652 \\
\hline \multirow{2}{*}{ Center Street and Hester Street } & 3 & 1 & 16 & 1250 \\
\hline & 1 & 1 & 17 & 379 \\
\hline \multirow{2}{*}{ Lafayette Street and Walker Street } & 2 & 1 & 14 & 889 \\
\hline & 1 & 1 & 16 & 443 \\
\hline \multirow{2}{*}{ Broadway and Franklin Street } & 2 & 1 & 14 & 893 \\
\hline & 1 & 1 & 16 & 448 \\
\hline \multirow{2}{*}{ Church Street and Walker Street } & 3 & 1 & 16 & 1258 \\
\hline & 1 & 1 & 16 & 438 \\
\hline
\end{tabular}

Input: Highway Network $H_{c}$ with full capacity $c a_{j}$ for the intersection $a_{j} \in V$, and $c b_{j}$ for the intersection

$b_{j} \in V$, facility class $f$ class $_{j}$, number of lanes $n a_{j}, n b_{j}$ for each link $j \in E$. Capacity Loss Prediction

Model $\mathrm{Cl}$. Set of adjacent links links $s_{i}$, number of conflicts conflict $_{i}$, number of links connected to

the node local $_{i}$ for each intersection $i \in V$.

Output: Table $H r$ with unsignalized capacity $c r a_{j}$ towards intersection $a_{j} \in V$, and $c r b_{j}$ towards intersection $b_{j} \in V$.

$H r \leftarrow H_{c} ;$

For each intersection $i$ do

iflocal $_{i} \geq 3$ do

For each link $k$ in links $_{i}$ do

if $i=a_{k}$ and $n a_{k}>0$ do

$\operatorname{cra}_{k} \leftarrow \operatorname{Cl}\left(n a_{j}\right.$, conflict $_{i}$, fclass $\left._{k}\right) ;$

$c a_{k} \leftarrow \max \left(c r a_{k}, 100\right) ;$

if $i=b_{k}$ and $n b_{k}>0$ do

$c r b_{k} \leftarrow \operatorname{Cl}\left(n b_{j}, \operatorname{conflict}_{i}\right.$, fclass $\left._{k}\right) ;$

$c b_{k} \leftarrow \max \left(c r b_{k}, 100\right)$;

else

return $\mathrm{Hr}$;

FIgURE 5: Pseudocode of prediction and assignment of capacity loss.

2.4. Proposed Scenarios. The case study includes six capacityloss scenarios, along with base scenario without capacity loss. These scenarios, namely, Scenarios 1 to 6, simulate hypothetical power loss of six NTAs: East Village, West Village, Soho-TriBeCa-Civic Center-Little Italy, Battery Park City-Lower Manhattan, Chinatown, and Lower East Side, respectively. Study area is shown in Figure 6. In each scenario, during the hurricane evacuation process, power loss is assumed to occur in one of these NTAs and it is assumed to cause failures of all signals in the respective NTA. The length of all power-loss scenarios is assumed to be 12 hours, from midday to midnight.

For all scenarios, the same travel demands are applied using the same methodology proposed in previous research 2 , where the evacuation demand is identified by calculating the evacuation population in each census tract, then 


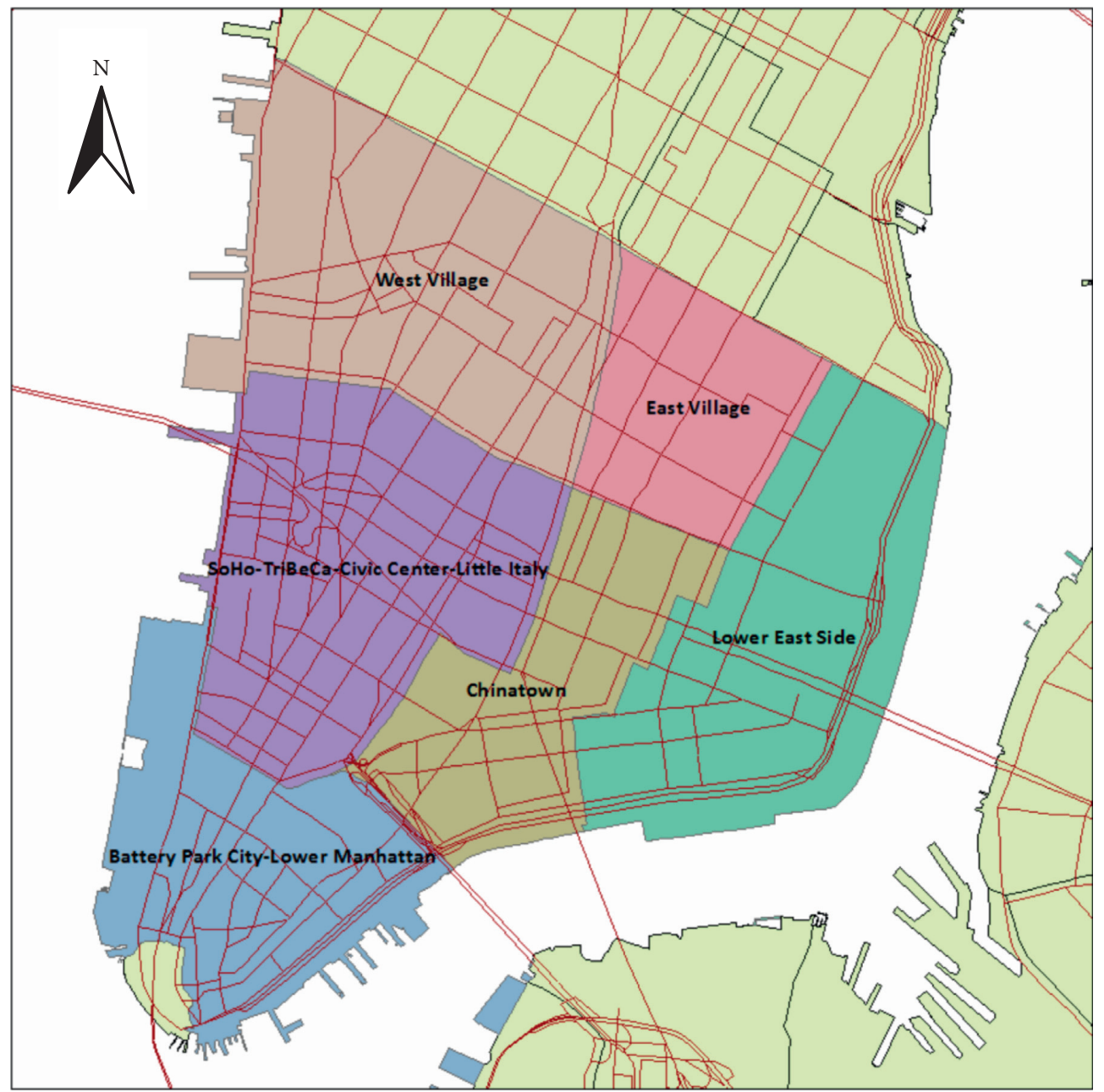

Figure 6: The study area.

combining them to generate evacuation O-D matrix in terms of TAZs (traffic analysis zone used in the planning model). Then, the evacuation traffic is split into hours using evacuation response curve. The curve is estimated using traffic counts from Transportation Operations Coordination Committee (TRANSCOM). Because evacuation only takes small proportion of total traffic demand, background traffic is calculated using the empirical trip table and excluding the trips towards vulnerable zones. The hourly traffic demand is the sum of evacuation and background demand. The simplified demand modeling approach is depicted in Figure 7.

By comparing the network performance, the severity of cascading failures can be evaluated. The evaluation considered metrics related to OD travel time, speed, and volume during the study periods among neighborhoods, and the base-case scenario with no signal failures. The comparative results provide some insights into the resilience of the neighborhoods.

\section{Results}

The simulation was implemented through the NYBPM models, and the results in terms of the changes in travel times based on TAZs are shown in Figure 8. Travel times after the power loss are compared with the travel times in the base scenario, i.e., travel times without any capacity losses. The average travel time of each TAZ is calculated using travel times of all the trips leaving a specific TAZ whose destinations are within Manhattan. Longer interborough trips were eliminated for the sake of avoiding bias.

It can be shown that in all six scenarios, signal failures lead to an increase of travel times of the neighborhood, such impacts which are alleviated for further zones. For Scenario 1 (East Village) and Scenario 2 (West Village), travel times for TAZs within these NTAs are increased by $20 \%$ and less than $10 \%$ percent for other surrounding TAZs. For Scenario 3 of Soho-TriBeCa-Civil Center-Little Italy (STCL), travel times are increased by $20 \%$ to $30 \%$. For Scenario 4 (Battery Park City-Lower Manhattan), the travel times are more than doubled in the southern tip of Financial District. Moderate increase of travel times is observed in a small number of TAZs within Scenario 5 (Chinatown) and Scenario 6 (Lower East Side), and its impact is negligible for surrounding neighborhoods compared to other scenarios.

Figure 9 visualizes the changes in link volumes compared to the based scenario, together with Figure 10 comparing differences of link speeds. It can be found that the 12-hour 


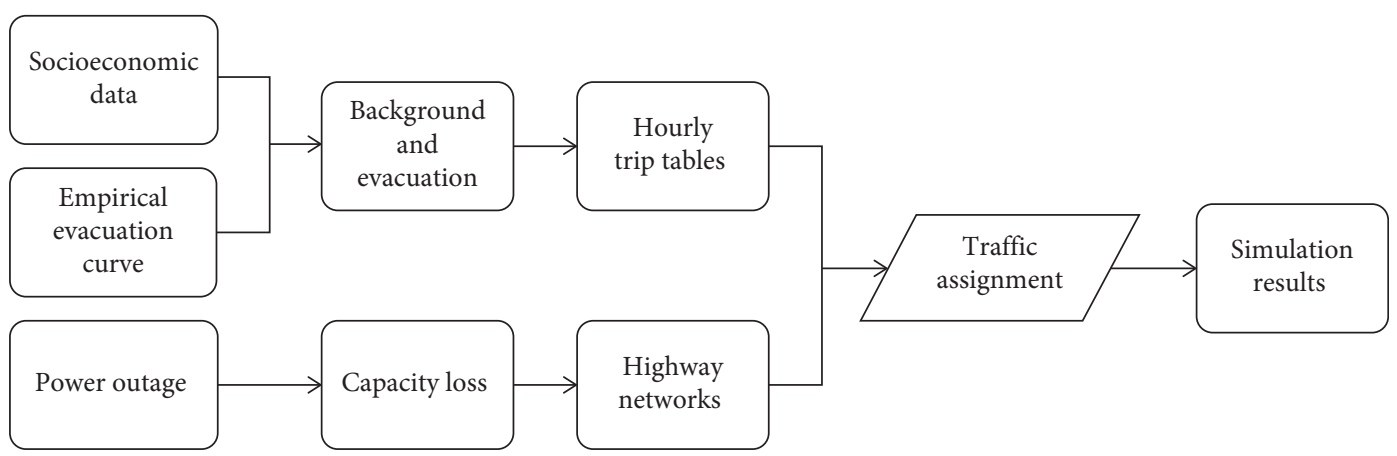

FIgURE 7: Demand modeling flowchart.

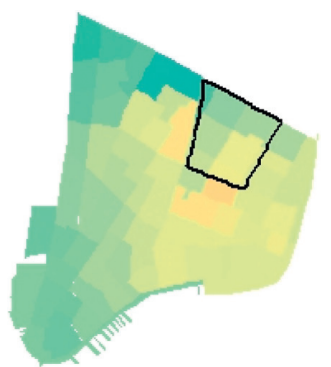

(a)

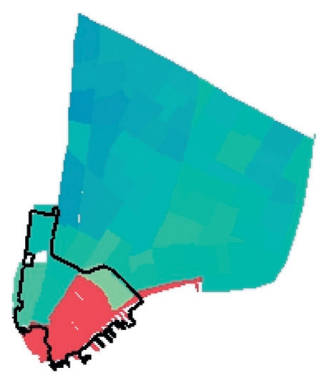

(d)

$$
\begin{aligned}
& \text { Range } \\
& \text { — Power loss }
\end{aligned}
$$

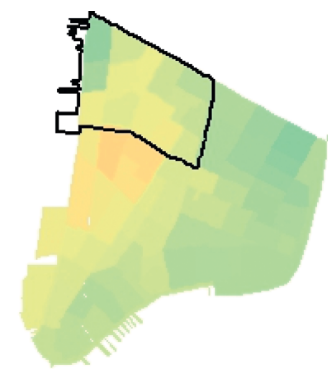

(b)

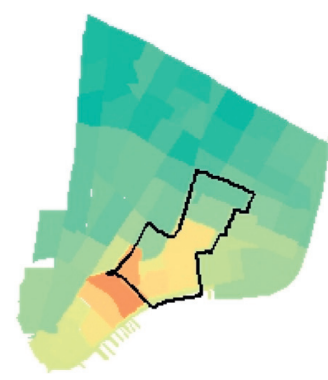

(e)

Difference of travel times

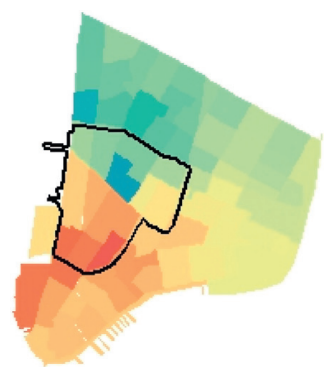

(c)

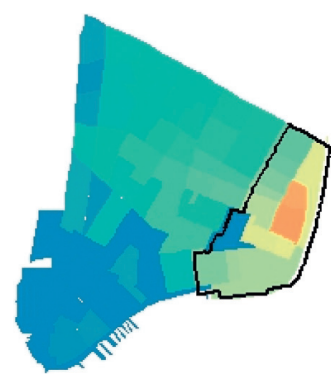

(f)

Figure 8: Changes in average travel times by TAZs. (a) Scenario 1: East Village. (b) Scenario 2: West Village. (c) Scenario 3: SoHo-TriBeCaCivic Center-Little Italy. (d) Scenario 4: Battery Park City-Lower Manhattan. (e) Scenario 5: Chinatown. (f) Scenario 6: Lower East Side.

volumes of roads within power-loss areas are reduced significantly, as shown in the green links for each scenario. The excessive traffic is diverted to the neighboring areas and causes different extents of volume increase. For Scenarios 1 and 2, although the volume and speed changes within powerloss zones are observable, the signal failures do not have a strong impact on neighboring zones. For Scenario 3, however, the capacity loss of STCL area diverts a considerable amount of traffic to Chinatown and Lower East Side and deteriorates the performance of nearly the entire network. This is probably because the critical corridors within STCL, such as Canal Street and the Holland Tunnel, are disrupted, causing congestion. Compared with Scenario 3, impact area of Scenario 4 is not as significant, but a significant amount of performance reduction is observed, especially the amount of traffic diverted to Chinatown. This should be attributed to degraded operation of the Battery Tunnel, and traffic is diverted to nearby Brooklyn and Manhattan Bridges with entrances located in Chinatown. This can also explain the network-level speed reduction in Scenario 5. For Scenario 6, the impact of signal loss is not significant and only the links near the Williamsburg Bridge are primarily affected.

Apart from measures of average performance, the variation of travel times within the study periods is evaluated for each neighborhood, as displayed in Figure 11. For all six capacity-loss scenarios, the travel times are higher than the base scenario. Such differences are higher in the first hours of evacuation period and become lower with reduction of travel demands. It can be observed that Scenario 3 causes the most significant increases of travel times for all neighborhoods. 


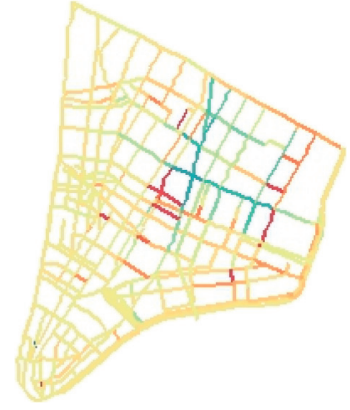

(a)

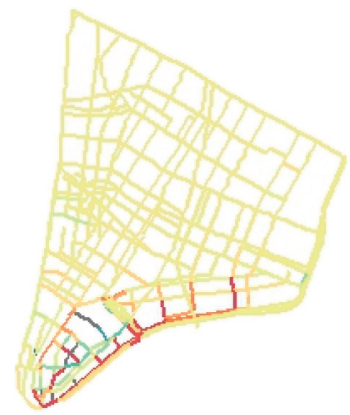

(d)

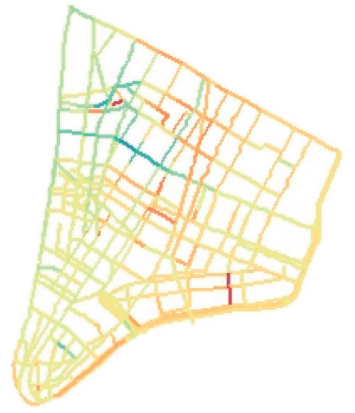

(b)

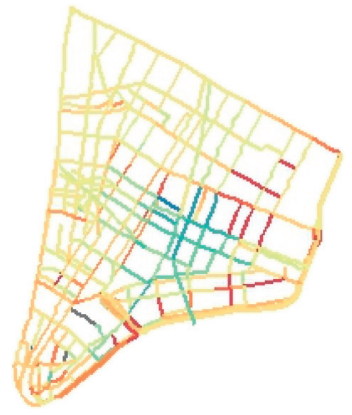

(e)

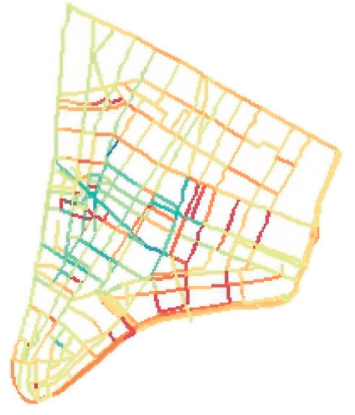

(c)

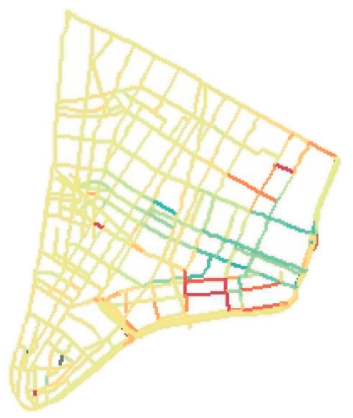

(f)

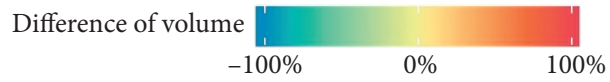

Figure 9: Changes in link volumes. (a) Scenario 1. (b) Scenario 2. (c) Scenario 3. (d) Scenario 4. (e) Scenario 5. (f) Scenario 6.

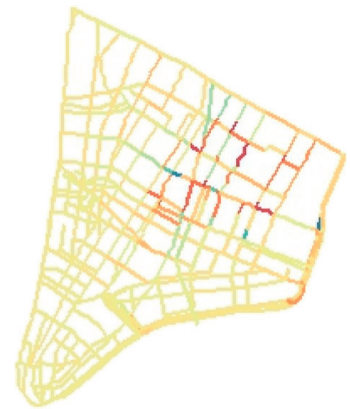

(a)

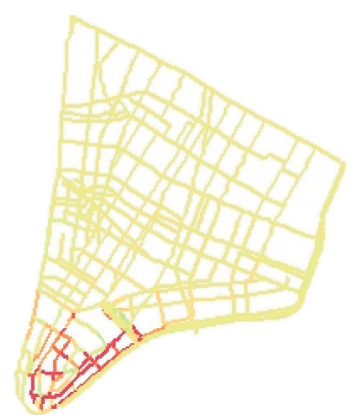

(d)

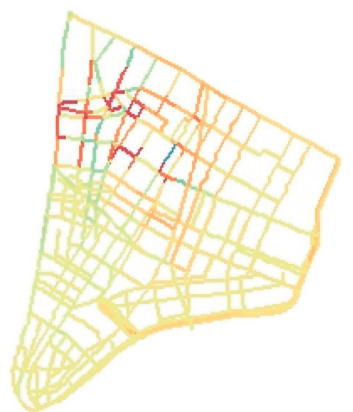

(b)

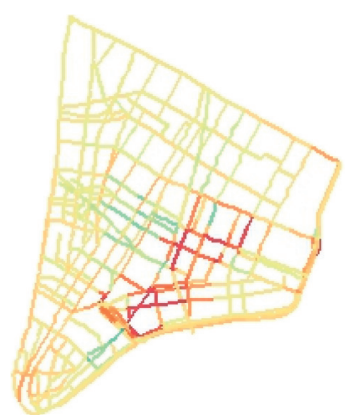

(e)

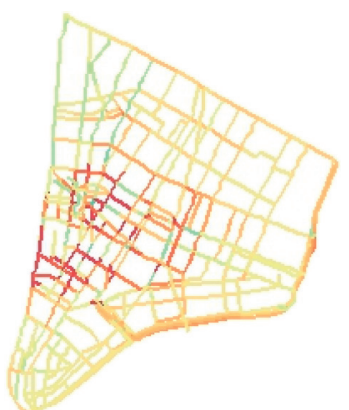

(c)

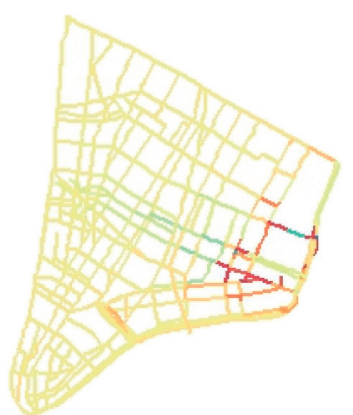

(f)

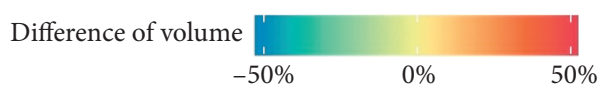

Figure 10: Differences in link speeds. (a) Scenario 1. (b) Scenario 2. (c) Scenario 3. (d) Scenario 4. (e) Scenario 5 . (f) Scenario 6. 


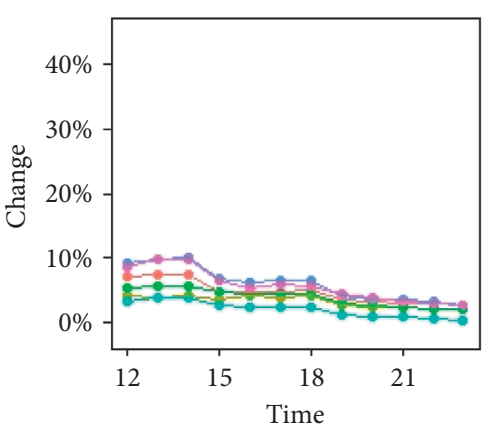

(a)

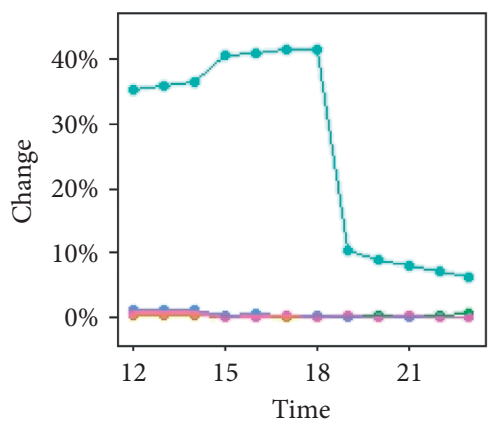

(d)

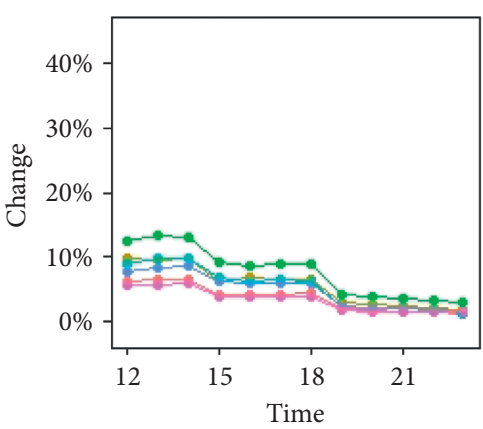

(b)

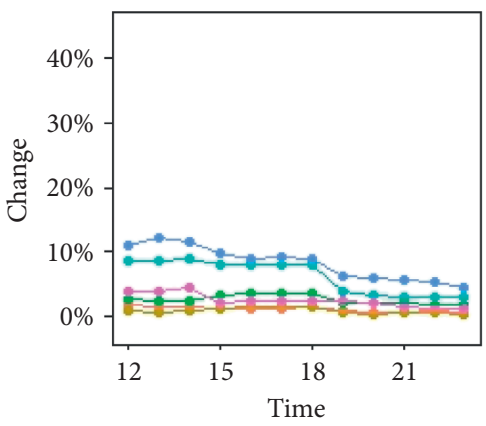

(e)

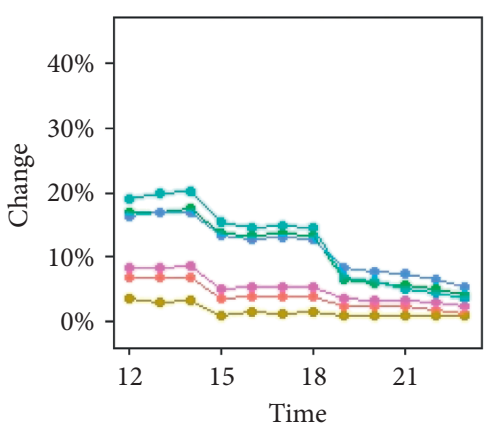

(c)

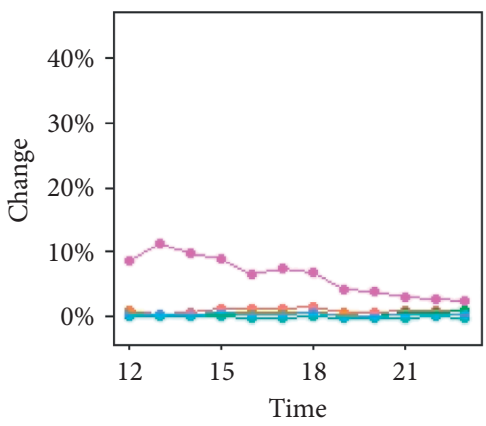

(f)

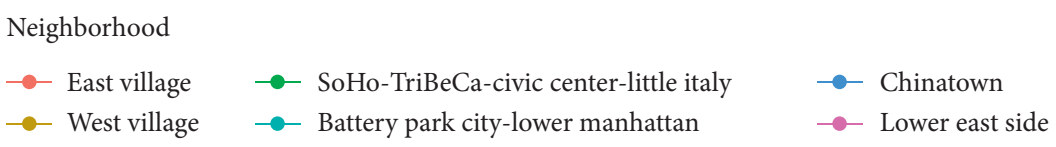

Figure 11: Variation of travel times for neighborhoods. (a) Scenario 1. (b) Scenario 2. (c) Scenario 3. (d) Scenario 4 . (e) Scenario 5. (f) Scenario 6.

For Scenarios 4 and 6, the capacity loss affects its own neighborhood rather than neighboring zones. The impacts of Scenarios 1, 2, and 5 on the network are close and also follow the temporal patterns. The impact of Scenario 6 is the lowest. These results are in agreement with the other findings above.

The results above show that when the signal failures happen in STCL, southern tip of Battery Park, and the eastmost of TAZs in Lower East Sides neighborhood, they lead to considerable degrading of roadway performance. This result implies that if the signal failure occurred next to major roadway links used for evacuation, such as entrances of critical bridges and tunnels, then their capacity will be reduced significantly. This is due to the large traffic demand and missing of signal controls for major intersections located in these areas. These results provide an insight into the prehurricane resilience for different neighborhoods located in lower Manhattan, which can be used to infer that roadway systems in the East Village, West Village, and Lower East Side are more resilient and can maintain a more stable level of service compared to neighborhoods such as STCL and Battery Park during evacuation under signal failures.

\section{Conclusions}

This paper discussed the cascading failures of signal systems due to the failure of power-supply infrastructure. Based on the existing literature and common practice, signalized intersections losing power were treated as AWSC. Differences in intersection capacity are modeled and calibrated based on the results obtained from microscopic intersection models.

A scenario-based macroscopic model was built to simulate its impacts on the transportation network and evacuation process. Six evacuation scenarios with the same evacuation demand and signal failures at different locations were built. Besides, spatial-temporal patterns of zonal and link performances were evaluated. It can be concluded that the impact of signal failures can have a strong influence on evacuation efficiency for both the area where power is lost and its surrounding areas. Also, the significance of the impact is related to the intensity of evacuation demand, density of traffic signals, adjacency to the major roadway links, and interconnectivity to neighboring zones.

The model proposed in this approach can provide insight for government agencies and planners of transportation system resilience. For example, the model can show the vulnerable intersection or roadway segments and evaluate the impact of signal failures during the evacuation process, so the government agencies can locate the priority area when taking actions to guarantee the traffic operation. In addition, considering the different levels of resilience, the model can help the decision makers to identify the evacuation destinations and routes in which the traffic impact by the power outage is minimal. 
In future study, the proposed unsignalized capacityloss model can be improved by conducting SUMO analyses using additional combinations of physical geometries and mixed traffic conditions. Besides traffic signals, the cascading failures of the power system can also include malfunction of the public transit system, particularly subway systems. Another possible direction is to evaluate additional demands diverted from public transit to the road network due to the suspension of the subway network.

\section{Data Availability}

There are no linked research data sets for this submission. The following reason is given: the authors do not have permission to share data.

\section{Disclosure}

An early version of this manuscript was presented in Transportation Research Board 98th Annual Meeting of Transportation Research Board.

\section{Conflicts of Interest}

The authors declare that they have no conflicts of interest.

\section{Authors' Contributions}

The authors confirm contribution to the paper as follows: Y. Zhu, H. Yang, and K. Ozbay were responsible for study conception and design. Y. Zhu, F. Zuo, and D. Sha collected data. Y. Zhu and H. Yang carried out analysis and interpretation of results. Y. Zhu, K. Ozbay, and H. Yang did draft manuscript preparation. All the authors reviewed the results and approved the final version of the manuscript.

\section{Acknowledgments}

This study was partially supported by the Young Scientists Fund of the National Natural Science Foundation of China (grant no. 61903205) and Young Scientists Fund of Natural Science of Inner Mongolia (grant no. 2019BS07002). This study was partially support by the project of the NSF CRISP: Type 1: Reductionist and Integrative Approaches to Improve the Resiliency of Multi-Scale Interdependent Critical Infrastructure, and the project on A Decision-Support System for Resilient Transportation Networks funded by NYU Provost Global Seed Fund Grants.

\section{References}

[1] J. Li and K. Ozbay, "Evacuation planning with endogenous transportation network degradations: a stochastic cell-based model and solution procedure," Networks and Spatial Economics, vol. 15, no. 3, pp. 677-696, 2014.

[2] Y. Zhu, K. Ozbay, K. Xie, H. Yang, and E. F. Morgul, "Network modeling of hurricane evacuation using data driven demand and incident induced capacity loss models," in Proceedings of the 2016 Transportation Research Board, Washington, D.C., USA, 2016.
[3] K. Xie, K. Ozbay, and H. Yang, "Spatial analysis of highway incident durations in the context of Hurricane Sandy," Accident Analysis \& Prevention, vol. 74, pp. 77-86, 2015.

[4] H. Fotouhi, S. Moryadee, and E. Miller-Hooks, "Quantifying the resilience of an urban traffic-electric power coupled system," Reliability Engineering \& System Safety, vol. 163, pp. 79-94, 2017.

[5] L. Zhang, B.-B. Fu, and Y.-X. Li, "Cascading failure of urban weighted public transit network under single station happening emergency," Procedia Engineering, vol. 137, pp. 259266, 2016.

[6] V. Oricchio, M. P. Hunter, and D. Jared, "Microscopic simulation model of traffic operations at intersections in malfunction flash mode," Transportation Research Record: Journal of the Transportation Research Board, vol. 2080, no. 1, pp. 67-74, 2008.

[7] H. Wegmann, "A general capacity formula for unsignalized intersections," Intersections Without Traffic Signals II, Springer, New York, NY, USA, pp. 177-191, 1991.

[8] K. G. Baass, "The potential capacity of unsignalized intersections," ITE Journal, vol. 57, no. 10, pp. 43-46, 1987.

[9] W. Brilon and T. Miltner, "Capacity at intersections without traffic signals," Transportation Research Record: Journal of the Transportation Research Board, vol. 1920, no. 1, pp. 32-40, 2005.

[10] J. Prasetijo, M. H. Pour, and S. M. R. Ghadiri, "Capacity of unsignalized intersections under mixed traffic conditions," Procedia-Social and Behavioral Sciences, vol. 16, pp. 676-685, 2011.

[11] S. I. Guler and M. Menendez, "Methodology for estimating capacity and vehicle delays at unsignalized multimodal intersections," International Journal of Transportation Science and Technology, vol. 5, no. 4, pp. 257-267, 2016.

[12] New York Metropolitan Transportation Council, Best practice model, available online at: http://www.nymtc.org/project/ bpm/bpmindex.html.

[13] Caliper, TransCAD - transportation planning software, available online at: http://www.caliper.com/tcovu.htm.

[14] NYSDOT, Functional classification, available online at: https://www.dot.ny.gov/gisapps/functional-class-maps.

[15] M. Haklay and P. Weber, "Openstreetmap: user-generated street maps," IEEE Pervasive Computing, vol. 7, no. 4, pp. 12-18, 2008.

[16] M. Behrisch, L. Bieker, J. Erdmann, and D. Krajzewicz, "SUMO-simulation of urban mobility: an overview," in Proceedings of the SIMUL 2011, the Third International Conference on Advances in System Simulation, Barcelona, Spain, October 2011.

[17] D. Husch and J. Albeck, Trafficware Synchro 6 User Guide, TrafficWare, Albany, CA, USA, 2004.

[18] H. C. Manual, HCM2010. Transportation Research Board, National Research Council, Washington, D.C., USA, 2010. 